\title{
Performance of similarity explicit group iteration for solving 2D unsteady convection-diffusion equation
}

\author{
Nur Afza Mat Ali ${ }^{1}$, Jumat Sulaiman², Azali Saudi ${ }^{3}$, Nor Syahida Mohamad ${ }^{4}$ \\ ${ }^{1,2,4}$ Faculty of Science and Natural Resources, Universiti Malaysia Sabah (UMS), Malaysia \\ ${ }^{3}$ Faculty of Computing and Informatics, Universiti Malaysia Sabah (UMS), Malaysia
}

\begin{tabular}{l} 
Article Info \\
\hline Article history: \\
Received Mar 31, 2021 \\
Revised Jun 8, 2021 \\
Accepted Jun 17, 2021 \\
\hline Keywords: \\
Convection-diffusion equation \\
Partial differential equation \\
Similarity explicit group \\
Similarity finite difference \\
Similarity solution
\end{tabular}

\section{Corresponding Author:}

Jumat Sulaiman

Faculty of Science and Natural Resources

Universiti Malaysia Sabah

Jalan UMS, 88400 Kota Kinabalu, Sabah, Malaysia

Email: jumat@ums.edu.my

\begin{abstract}
In this paper, a similarity finite difference (SFD) solution is addressed for the two-dimensional (2D) parabolic partial differential equation (PDE), specifically on the unsteady convection-diffusion problem. Structuring the similarity transformation using wave variables, we reduce the parabolic PDE into elliptic PDE. The numerical solution of the corresponding similarity equation is obtained using a second-order central SFD discretization scheme to get the second-order SFD approximation equation. We propose a fourpoint similarity explicit group (4-point SEG) iterative method as a numerical solution of the large-scale and sparse linear systems derived from SFD discretization of 2D unsteady convection-diffusion equation (CDE). To show the 4-point SEG iteration efficiency, two iterative methods, such as Jacobi and Gauss-Seidel (GS) iterations, are also considered. The numerical experiments are carried out using three different problems to illustrate our proposed iterative method's performance. Finally, the numerical results showed that our proposed iterative method is more efficient than the Jacobi and GS iterations in terms of iteration number and execution time.
\end{abstract}

This is an open access article under the CC BY-SA license.

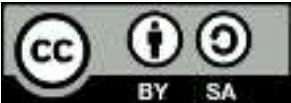

\section{INTRODUCTION}

Convection-diffusion equation (CDE) is one of the most challenging problems and frequently used in various branches of engineering and applied science, especially in radial transport in a porous medium [1], heat transfer in a nanofluid filled [2], heat transfer in a draining film [3], and water transport in soil [4]. Also, the applications of CDE can be found in [5]-[8]. Due to its application, these problems have received extensive attention, and many researchers attempted to solve these problems numerically to achieve the lowest computational complexity and highest performance. To achieve the low computational complexity, there are many studies on similarity solution techniques have been explored by many researchers and applied in partial differential equations (PDEs). For instance, Afify [9] presented similarity solutions in magnetohydrodynamic, which is obtained by using scaling transformations then solved numerically by using the shooting technique with fourth-order Runge-Kutta integration scheme. M. Siavashi et al. [10], the authors obtained a similarity solution of air and nanofluid impingement cooling of a cylindrical porous heat sink using similarity variables then solved numerically. Usman et al. [11], a similarity solution of the water/magnetite nanofluid modelled PDEs subject to thermal radiation and Lorentz force over stretchable rotating disks is obtained by supporting precise similarity transformation. The studies on the similarity 
solution can also be found in [12]-[15]. Basically, the main idea of using a similarity solution is to reduce the number of independent variables of a system of PDEs at least one less than that of the original equation. Because of the low computational complexity and high computational performance of this method, its implementation is of interest.

Many researchers have solved the CDE using the finite difference method (FDM) in addition to the above numerical methods for solving the proposed CDE problem as noted in the first paragraph. As a result, a new finite difference (FD) discretization scheme has been proposed by combining several numerical discretization schemes, mostly from the FD scheme family. For instance, the nonstandard FD [16]-[18], the exponential FD [19]-[21] and perturbational FD [22] are implemented for solving CDE. No doubt that [16]-[22] can present outstanding performance, but it suffers from high computational complexity because the obtained approximation equation generated a sequence of linear systems for each time level, in which this phenomenon will increase computational complexity. The computational complexity of algorithms is determined by two norms: the number of nodes that have to be examined and the operational cost per node. The more linear systems, the more nodes will be used that will be affected by computational complexity. In conjunction with new FD discretization schemes in [16]-[22], we aim to introduce an entirely new FD scheme based on combination of the similarity and FD schemes, named as similarity finite difference (SFD) discretization schemes, to investigate its feasibility in solving CDE.

Based on the second paragraph, we can see that many researchers have proposed a new FD discretization scheme with getting high computational performance and lower computational complexity. Apart from that, we attempt to examine the feasibility of the SFD discretization scheme as a numerical method to solve 2D unsteady CDE. Based on the previous literature review on applying the similarity solutions in the first paragraph, the findings showed that the similarity solution technique has successfully reduced the independent variables of PDEs and then transform them into ordinary differential equation (ODE). It means that the computational cost of the similarity technique will decrease and make the computational complexity of the similarity approximation equation low compared to without using similarity solutions. However, most of them are solving PDEs using similarity solutions are concerned with PDE problems being reduced to ODE problems, but the studies concerning reducing parabolic PDEs into the corresponding elliptic PDEs have not been found yet. The main idea is to maintain the dimension of the model so that the characteristic of the object can be preserved. Inspired by this technique's low computational complexity and high computational performance, we propose newly SFD discretization schemes. With that, we applied the similarity solution specifically using wave variables transformation in order to reduce 2D parabolic PDEs in details 2D unsteady CDE problem into 2D elliptic PDEs.

The 2D unsteady CDE is first transformed into a 2D elliptic PDE via a similarity solution technique to solve the proposed problem. The SFD approximation equation is then formed by discretizing the corresponding elliptic PDE using the newly SFD discretization schemes. Since the SFD approximation equation produces a large-scale and sparse linear system with its matrix coefficient, an effective solver must solve the resulting large and sparse linear system. According to the results in [23]-[25], the iterative approach is most definitely the best linear solver for large and sparse linear systems. Several iterative methods for solving a linear system with a large-scale and sparse coefficient matrix are discussed in the literature. The implementations of the point iteration family, such as successive over-relaxation (SOR) [26], [27], accelerated over-relaxation (AOR) [28], [29], and kaudd successive over-relaxation (KSOR) [30], [31], can be used to solve this linear method. Evans [32] invented the explicit group (EG) iteration method for obtaining an approximate solution by grouping the linear system into a sequence of $(4 \times 4)$ linear systems based on the coefficient matrix's characteristics. Compared to the GS iteration, this block iterative method is faster. Although the convergence rate for EG iteration has been accelerated, several researchers have created new versions of the EG iteration family, including 4EGSOR via nine-point Laplacian (4EGSOR9L) [33], 4 point-explicit decoupled group (EDG) [34] and 9 Point-EDGSOR [35] in which all of these block iterations have significantly decreased their convergence rate. Ali et al. [36] and Bee et al. [37] used the EG method for solving the 2D CDE to solve the linear system of unsteady advection-diffusion problem iteratively, and their results indicated that this method has a fast convergence due to the large number of points that must be treated simultaneously. However, without using similarity solution techniques, the high computational complexity will occur to get the approximate solution of the 2D unsteady CDE. To achieve the low computational complexity, we propose a new variant of the EG iteration family to solve 2D unsteady CDE by solving the generated linear system.

As a result, the remainder of this paper focuses on evaluating the efficacy of the four-point similarity explicit group (4-point SEG) iterative method for solving the system of SFD approximation equations, which was inspired by the newly developed SFD discretization scheme. The combination of the SFD scheme and the EG iterative method can be used to formulate the 4 point-SEG iterative method. To illustrate the feasibility of 4-point SEG iteration, the following general equation for 2D unsteady CDE is 
considered in this paper to investigate the feasibility of the SFD discretization schemes and performance of the 4 point-SEG iterative method:

$$
\frac{\partial u}{\partial t}+\beta_{1} \frac{\partial u}{\partial x}+\beta_{2} \frac{\partial u}{\partial y}=\alpha_{1} \frac{\partial^{2} u}{\partial x^{2}}+\alpha_{2} \frac{\partial^{2} u}{\partial y^{2}}+R(x, y, t), \Omega \times(0, T],
$$

subject to the following conditions

$$
\begin{aligned}
& u(x, y, t)=g(x, y, t),(x, y) \in \partial \Omega, t \in(0, T], \\
& u(x, y, 0)=h(x, y),(x, y) \in \Omega,
\end{aligned}
$$

where $\beta_{1}$ and $\beta_{2}$ are constant speeds of convection in the direction of $x$ and $y$ respectively; $\alpha_{1}>0$ and $\alpha_{2}>0$ are the coefficients of diffusivity in the $x-$ and $y-$ directions, respectively; $\Omega$ is a subset of $\mathbb{R}^{2}$; $(0, T]$ is the time interval; $g(x, y, t)$ and $h(x, y)$ denote the smooth functions.

\section{SFD APPROXIMATION EQUATION}

Before we start the discretization process of problem (1), firstly, we transform problem (1) into the 2D elliptic PDE using similarity transformation specifically on wave variables as explained in the previous section. To start the transformation process, let us consider the wave variables as follows [27], [38], [39];

$$
\begin{gathered}
\xi=x-c t, \\
\tau=y-d t
\end{gathered}
$$

and we use the transformation $u(x, y, t)=u(\xi, \tau)$. Using (3), in (1) reduces to an elliptic PDE as;

$$
\zeta\left(\frac{\partial u}{\partial \xi}+\frac{\partial u}{\partial \tau}\right)+\alpha\left(\frac{\partial^{2} u}{\partial \xi^{2}}+\frac{\partial^{2} u}{\partial \tau^{2}}\right)=-R(\xi, \tau), \Omega \in\left[\xi_{a}, \xi_{b}\right] \times\left[\tau_{a}, \tau_{b}\right]
$$

where $\zeta=\frac{\eta}{2}-\beta, \beta=\beta_{1}=\beta_{2}, \alpha=\alpha_{1}=\alpha_{2}$. Let us build the distribution of uniformly node points as shown in Figure 1 to help us explore the derivation of the similarity approximation equation.

From the Figure 1, we must discretize the solution domain, $(\Omega)$ uniformly in both $\xi$ and $\tau$ directions with a mesh size, $h$ which is defined as $\Delta \xi=\frac{\xi_{b}-\xi_{a}}{m}, \Delta \tau=\frac{\tau_{b}-\tau_{a}}{m}, h=\Delta \xi=\Delta \tau$ and $m=n+1$. Using the SFD scheme and the finite grid network in Figure 1, we discretize the 2D elliptic PDEs (4) as follows:

$$
\begin{aligned}
& \left.\frac{d u}{d \xi}\right|_{i j}=\frac{U_{i+1, j}-U_{i-1, j}}{2 h} \\
& \left.\frac{d^{2} u}{d \xi^{2}}\right|_{i j}=\frac{U_{i+1, j}-2 U_{i, j}+U_{i-1, j}}{h^{2}} \\
& \left.\frac{d u}{d \tau}\right|_{i j}=\frac{U_{i, j+1}-U_{i, j-1}}{2 h} \\
& \left.\frac{d^{2} u}{d \tau^{2}}\right|_{i j}=\frac{U_{i, j+1}-2 U_{i, j}+U_{i, j-1}}{h^{2}}
\end{aligned}
$$

Then substitute (5) into (4), we have the following approximation equation;

$$
\zeta\left(\frac{U_{i+1, j}-U_{i-1, j}}{2 h}+\frac{U_{i, j+1}-U_{i, j-1}}{2 h}\right)+\alpha\left(\frac{U_{i+1, j}-2 U_{i, j}+U_{i-1, j}}{h^{2}}+\frac{U_{i, j+1}-2 U_{i, j}+U_{i, j-1}}{h^{2}}\right)=-R_{i, j},
$$

By simplifying (6), we get;

$$
A U_{i-1, j}+B U_{i+1, j}+A U_{i, j-1}+B U_{i, j+1}-4 U_{i, j}=r_{i, j}
$$

where $A=\frac{\gamma_{1}-\gamma_{2}}{\gamma_{1}}, B=\frac{\gamma_{1}+\gamma_{2}}{\gamma_{1}}, \gamma_{1}=\frac{\alpha}{h^{2}}, \gamma_{2}=\frac{\zeta}{2 h}$ and $r_{i, j}=-\frac{R_{i, j}}{\gamma_{1}}$.

Based on the SFD approximation (7), a linear system generates with the coefficient matrix is large-scale and sparse in matrix form as (8) by taking $i=1,2,3, \ldots, n$

$$
F \underline{U}=\underline{r}
$$


where;

$$
F=\left[\begin{array}{cccccc}
G_{2} & G_{3} & 0 & 0 & \cdots & 0 \\
G_{1} & G_{2} & G_{3} & 0 & \cdots & 0 \\
0 & G_{1} & G_{2} & G_{3} & \cdots & 0 \\
\vdots & \vdots & \vdots & \vdots & \ddots & \vdots \\
0 & 0 & 0 & G_{1} & G_{2} & G_{3} \\
0 & 0 & 0 & 0 & G_{1} & G_{2}
\end{array}\right], \underline{U}=\left[\begin{array}{c}
\underline{U}_{1} \\
\underline{U}_{2} \\
\underline{U}_{3} \\
\underline{U}_{4} \\
\vdots \\
\underline{U}_{n}
\end{array}\right], \underline{r}=\left[\begin{array}{c}
\underline{r}_{1} \\
\underline{r}_{2} \\
\underline{r}_{3} \\
\underline{r}_{4} \\
\vdots \\
\underline{r}_{n}
\end{array}\right]
$$

and

$$
\begin{aligned}
G_{1} & =\left[\begin{array}{ccccc}
A & 0 & 0 & \cdots & 0 \\
0 & A & 0 & \cdots & 0 \\
0 & 0 & A & \cdots & 0 \\
\vdots & \vdots & \vdots & \ddots & \vdots \\
0 & 0 & 0 & 0 & A
\end{array}\right], G_{2}=\left[\begin{array}{ccccc}
-4 & B & 0 & \cdots & 0 \\
A & -4 & B & \cdots & 0 \\
0 & A & -4 & \cdots & 0 \\
\vdots & \vdots & \vdots & \ddots & \vdots \\
0 & 0 & 0 & A & -4
\end{array}\right], G_{3}=\left[\begin{array}{ccccc}
B & 0 & 0 & \cdots & 0 \\
0 & B & 0 & \cdots & 0 \\
0 & 0 & B & \cdots & 0 \\
\vdots & \vdots & \vdots & \ddots & \vdots \\
0 & 0 & 0 & 0 & B
\end{array}\right], \\
\underline{U}_{i} & =\left[\begin{array}{llllll}
U_{1, i} & U_{2, i} & U_{3, i} & U_{4, i} & \cdots & U_{n, i}
\end{array}\right]^{T},
\end{aligned}
$$

for $i=1,2,3, \ldots, n$.

\section{DERIVATION OF 4-POINT SEG ITERATION}

Since the coefficient matrix for the linear system (8) has large-scale and spare characteristics, this study proposed a faster numerical solver by employing a 4-point SEG iteration. Now, in this section, the formulation of 4 point-SEG attempts to be established. To obtain the formulation of 4-point SEG, we consider the grid network in Figure 1 and a group of block node points concept in Figure 2 The finite grid network of the SFD approximation equation is depicted in Figure 2, where the block iteration approach has been made until iteration convergence is achieved.

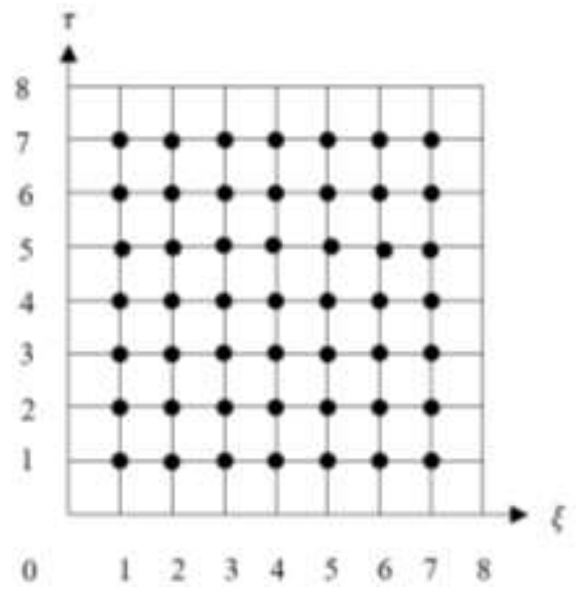

Figure 1. Finite grid networks at $m=8$

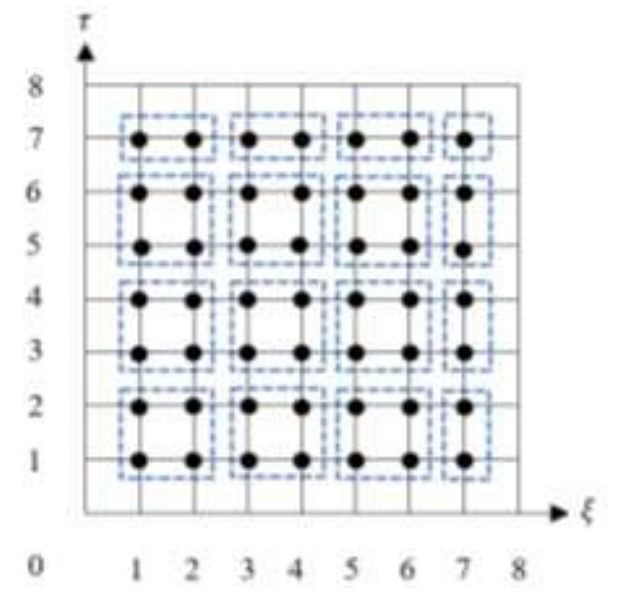

Figure 2. Implementation of the 4-point SEG iteration at solution domain, $\Omega$

Apply (7) on any group of four node points in the solution domain, the 4-point SEG iteration scheme can be formulated as;

$$
\left[\begin{array}{cccc}
4 & -B & 0 & -B \\
-A & 4 & -B & 0 \\
0 & -A & 4 & -A \\
-A & 0 & -B & 4
\end{array}\right]\left[\begin{array}{c}
u_{i, j} \\
u_{i+1, j} \\
u_{i+1, j+1} \\
u_{i, j+1}
\end{array}\right]=\left[\begin{array}{c}
s 1 \\
s 2 \\
s 3 \\
s 4
\end{array}\right]
$$

where,

$$
\begin{array}{ll}
s 1=A U_{i-1, j}+A U_{i, j-1}-r_{i, j}, & s 2=B U_{i+2, j}+A U_{i+1, j-1}-r_{i+1, j} \\
s 3=B U_{i+2, j+1}+B U_{i+1, j+2}-r_{i+1, j+1}, & s 4=A U_{i-1, j+1}+B U_{i, j+2}-r_{i, j+1}
\end{array}
$$


By establishing the inverse matrix of the coefficient system (9) and manipulating it, the general scheme of the 4-point SEG iteration can be written as;

$$
\left[\begin{array}{c}
u_{i, j} \\
u_{i+1, j} \\
u_{i+1, j+1} \\
u_{i, j+1}
\end{array}\right]^{(k+1)}=\frac{1}{\operatorname{det} 4}\left[\begin{array}{c}
c 1 s 1+c 3 s 2+c 6 s 3+c 3 s 4 \\
c 2 s 1+c 1 s 2+c 3 s 3+c 4 s 4 \\
c 5 s 1+c 2 s 2+c 1 s 3+c 2 s 4 \\
c 2 s 1+c 4 s 2+c 3 s 3+c 1 s 4
\end{array}\right]
$$

where $\operatorname{det} 4=8(4-A B), c 1=8-A B, c 2=2 A, c 3=2 B, c 4=A B, c 5=A^{2}$ and $c 6=B^{2}$. The 4-point SEG iteration which has been used to solve the proposed problem (1), is summarised in Algorithm 1.

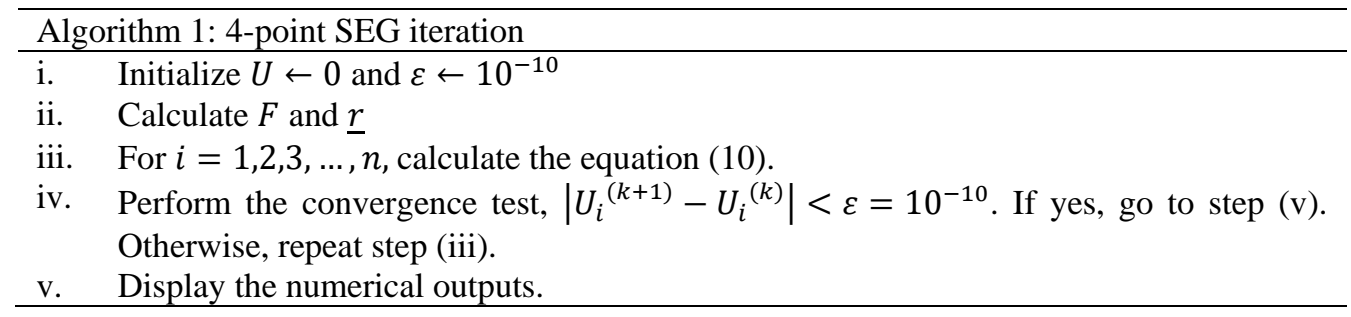

\section{NUMERICAL EXPERIMENT}

Three selected numerical experiments were performed in this section to illustrate the feasibility of 4-point SEG iteration in solving the 2D unsteady CDE (1) as compared with the Jacobi and GS iterations. For the sake of comparison, we considered three criteria include iteration number (Iter.), execution time in second (Time) and maximum absolute error (Err.). All the numerical experiments were run with different mesh sizes $(m)$ such as 64, 128, 256, 512, and 1024 .

Problem 1 [40]

Consider (1) in the unit square domain $[0,1] \times[0,1]$ with diffusion coefficients $\alpha=0.05$, convection coefficients $\beta=0.8$ and an analytic solution is;

$$
u(x, y, t)=\frac{1}{1+4 t} \exp \left(-\frac{\left(x-\beta_{1} t-0.5\right)^{2}}{\alpha_{1}(1+4 t)}-\frac{\left(y-\beta_{2} t-0.5\right)^{2}}{\alpha_{2}(1+4 t)}\right) \text {. }
$$

Problem 2 [41]

To demonstrate the benefits of the 4-point SEG iteration, we created a new test problem with an analytic solution as follow;

$$
u(x, y, t)=\exp (-2 t) \cos (x) \sin (2 y)
$$

and the source term is;

$$
R(x, y, t)=\exp (-2 t)\left[\cos (x+2 y)+\cos (x)\left(\frac{1}{2} \sin (2 y)+\cos (2 y)\right)\right] .
$$

Consider (1) in $[0,1] \times[0,1]$ with diffusion coefficients $\alpha=0.5$, and convection coefficients $\beta=1.0$. Problem 3 [42]

In this problem, we consider (1) in the unit square domain $[0,1] \times[0,1]$ with diffusion coefficients $a=b=\alpha=1.0$, convection coefficients $\beta=64.0$ and the analytic solution is

$$
u(x, y, t)=e^{-\left(a^{3}+b^{3}\right) t} \sin (a x+b y)
$$

and the source term is;

$$
R(x, y, t)=(a \beta+b \beta) e^{-\left(a^{3}+b^{3}\right) t} \cos (a x+b y)
$$

All the numerical results for 4-point SEG iteration together with Jacobi and GS iterations in solving the above three problems were collected and tabulated in Tables 1, 2 and 3, respectively.

Based on Tables 1 to 3, obviously, it shows that our proposed iterative method, namely 4-point SEG iteration, gives tremendously improve in term of iteration number and execution time, which significantly has approximately reduced iteration number and execution time by $58.32-68.34 \%$ and $50.86-66.89 \%$ for 
Problem 1, 69.83-72.17\% and 64.55-71.89\% for Problem 2 and $74.43-79.33 \%$ and $69.44-73.53 \%$ for Problem 3 respectively. Also, for the maximum absolute error, all iterative methods show excellent agreement and close to the exact solution.

Table 1. Comparison of Iter., Time, and Err. for Jacobi, GS and 4-point SEG iterations of Problem 1

\begin{tabular}{ccccccc}
\hline & Method & $64 \times 64$ & $128 \times 128$ & $256 \times 256$ & $512 \times 512$ & $1024 \times 1024$ \\
\hline Iter. & Jacobi & 2236 & 6732 & 19017 & 49868 & 119729 \\
& GS & 1268 & 3919 & 11434 & 31146 & 78287 \\
\multirow{5}{*}{ Time } & 4-point SEG & 708 & 2242 & 6744 & 19038 & 49908 \\
& Jacobi & 1.48 & 4.79 & 38.20 & 391.40 & 3785.64 \\
& GS & 0.82 & 2.77 & 25.30 & 261.91 & 2628.67 \\
Err. & 4-point SEG & 0.49 & 1.76 & 16.91 & 179.43 & 1860.27 \\
& Jacobi & $1.505817 \mathrm{E}-05$ & $1.515919 \mathrm{E}-05$ & $1.537614 \mathrm{E}-05$ & $1.620934 \mathrm{E}-05$ & $1.927445 \mathrm{E}-05$ \\
& GS & $1.504651 \mathrm{E}-05$ & $1.511897 \mathrm{E}-05$ & $1.522844 \mathrm{E}-05$ & $1.565958 \mathrm{E}-05$ & $1.725004 \mathrm{E}-05$ \\
& 4-point SEG & $1.504112 \mathrm{E}-05$ & $1.509937 \mathrm{E}-05$ & $1.515330 \mathrm{E}-05$ & $1.537699 \mathrm{E}-05$ & $1.620543 \mathrm{E}-05$ \\
\hline
\end{tabular}

Table 2. Comparison of Iter., Time, and Err. for Jacobi, GS and 4-point SEG iterations of Problem 2

\begin{tabular}{|c|c|c|c|c|c|c|}
\hline & \multirow{2}{*}{ Method } & \multicolumn{5}{|c|}{ Mesh Size } \\
\hline & & $64 \times 64$ & $128 \times 128$ & $256 \times 256$ & $512 \times 512$ & $1024 \times 1024$ \\
\hline \multirow[t]{3}{*}{ Iter. } & Jacobi & 9906 & 35147 & 122640 & 418746 & 1387700 \\
\hline & GS & 5231 & 18688 & 65793 & 227295 & 765604 \\
\hline & 4-point SEG & 2757 & 9907 & 35143 & 122627 & 418714 \\
\hline \multirow[t]{3}{*}{ Time } & Jacobi & 5.94 & 22.20 & 247.69 & 3522.95 & 44301.54 \\
\hline & GS & 3.14 & 12.52 & 145.05 & 1923.93 & 26991.47 \\
\hline & 4-point SEG & 1.67 & 7.45 & 86.06 & 1167.63 & 15703.74 \\
\hline \multirow[t]{3}{*}{ Err. } & Jacobi & $3.268668 \mathrm{E}-03$ & $3.269326 \mathrm{E}-03$ & $3.270326 \mathrm{E}-03$ & $3.273919 \mathrm{E}-03$ & $3.288190 \mathrm{E}-03$ \\
\hline & GS & $3.268631 \mathrm{E}-03$ & $3.269176 \mathrm{E}-03$ & $3.269730 \mathrm{E}-03$ & $3.271549 \mathrm{E}-03$ & $3.278696 \mathrm{E}-03$ \\
\hline & 4-point SEG & $3.268612 \mathrm{E}-03$ & $3.269102 \mathrm{E}-03$ & $3.269434 \mathrm{E}-03$ & $3.270365 \mathrm{E}-03$ & $3.273953 \mathrm{E}-03$ \\
\hline
\end{tabular}

Table 3. Comparison of Iter., Time, and Err. for Jacobi, GS and 4-point SEG iterations of Problem 3

\begin{tabular}{ccccccc}
\hline & Method & $64 \times 64$ & $128 \times 128$ & $256 \times 256$ & $512 \times 512$ & $1024 \times 1024$ \\
\hline Iter. & Jacobi & 421 & 1697 & 6611 & 25519 & 98073 \\
& GS & 158 & 750 & 3138 & 12552 & 49152 \\
& 4-point SEG & 87 & 387 & 1604 & 6403 & 25082 \\
Time & Jacobi & 0.34 & 1.23 & 14.90 & 200.16 & 3082.46 \\
& GS & 0.13 & 0.62 & 7.18 & 107.98 & 1657.81 \\
& 4-point SEG & 0.09 & 0.33 & 4.01 & 61.16 & 932.81 \\
Err. & Jacobi & $1.147132 \mathrm{E}-03$ & $1.138314 \mathrm{E}-03$ & $1.136067 \mathrm{E}-03$ & $1.135537 \mathrm{E}-03$ & $1.135553 \mathrm{E}-03$ \\
& GS & $1.147132 \mathrm{E}-03$ & $1.138313 \mathrm{E}-03$ & $1.136061 \mathrm{E}-03$ & $1.135513 \mathrm{E}-03$ & $1.135449 \mathrm{E}-03$ \\
& 4-point SEG & $1.147132 \mathrm{E}-03$ & $1.138312 \mathrm{E}-03$ & $1.136058 \mathrm{E}-03$ & $1.135501 \mathrm{E}-03$ & $1.135397 \mathrm{E}-03$ \\
\hline
\end{tabular}

\section{CONCLUSION}

In this paper, we have been successfully reduced 2D parabolic PDEs, particularly on the convection-diffusion problem, into 2D elliptic PDEs using the similarity solutions technique via wave variables in which we manage to get a low computational complexity as desired in this study. The similarity approximation equation has been discretizing by using the SFD discretization scheme to get the SFD approximation equation. This approximation equation generated a large-scale and sparse linear system then solved using 4-point SEG, GS and Jacobi iterations. The 4-point SEG iteration has achieved the highest performance based on the implementation of these three iterations since the iteration number and execution time was smaller than Jacobi and GS iterations. Thus, we can conclude that our proposed method is more efficient than GS and Jacobi iterations. This research will be further expanded into the use of SFD discretization scheme via half-sweep and quarter- sweep iteration families for solving the $2 \mathrm{D}$ unsteady CDE.

\section{ACKNOWLEDGEMENTS}

For the completion of this paper, the authors appreciate the fund obtained from Universiti Malaysia Sabah, Malaysia under the research grant scheme (GUG0491-1/2020). 


\section{REFERENCES}

[1] E. J. Veling, "Radial Transport in a Porous Medium with Dirichlet, Neumann and Robin-Type Inhomogeneous Boundary Values and General Initial Data: Analytical Solution and Evaluation," Journal of Engineering Mathematics, vol. 75, pp. 173-189, 2012, doi: 10.1007/s10665-011-9509-x.

[2] M. Sheikholeslami, R. Ellahi, and M. Hassan, "A Study of Natural Convection Heat Transfers in a Nanofuid Flled Enclosure with Elliptic Inner Cylinder," International Journal of Numerical Methods for Heat and Fluid Flow, vol. 24, no. 8, pp. 1906-1927, 2014, doi: 10.1108/HFF-07-2013-0225.

[3] J. Isenberg and C. Gutfnger, "Heat Transfer to a Draining Film," International Journal of Heat and Mass Transfer, vol. 16, no. 2, pp. 505-512, 1973, doi: 10.1016/0017-9310(73)90075-6.

[4] J. Y. Parlange, "Water Transport in Soils," Annual Review of Fluid Mechanics, vol. 12, pp. 77-102, 1980, doi: 10.1146/annurev.fl.12.010180.000453.

[5] P. A. García-Salaberri, "Modeling Diffusion and Convection in Thin Porous Transport Layers Using a Composite Continuum-Network Model: Application to Gas Diffusion Layers in Polymer Electrolyte Fuel Cells," International Journal of Heat and Mass Transfer, vol. 167, pp. 120824, 2021, doi: 10.1016/j.ijheatmasstransfer.2020.120824.

[6] C. Japhet, F. Nataf, and F. Rogler, "The Optimized Order 2 Method: Application to Convection-Diffusion Problems," Future Generation Computer Systems, vol. 18, no. 1, pp. 17-30, 2001, doi: 0.1016/S0167739X(00)00072-8.

[7] A. Bazile, E. Hachem, J. C. L. Huget, and Y. Mesri, "Variational Multiscale Error Estimator for Anisotropic Adaptive Fluid Mechanic Simulations: Application to Convection-Diffusion Problems," Computer Methods in Applied Mechanics and Engineering, vol. 331, pp. 94-115, 2018, doi: 10.1016/j.cma.2017.11.019.

[8] D. Shongsheng and P. Jianing, "Application of Convection-Diffusion Equation to the Analyses of Contamination Between Batches in Multi-Products Pipeline Transport," Applied Mathematics and Mechanics, vol. 19, no. 8, pp. 757-764, 1998.

[9] A. A. Afify, "Similarity Solution in MHD: Effects of Thermal Diffusion and Diffusion Thermo on Free Convective Heat and Mass Transfer over a Stretching Surface Considering Suction or Injection," Communications in Nonlinear Science and Numerical Simulation, vol. 14, no. 5, pp. 2202-2214, 2009, doi: 10.1016/j.cnsns.2008.07.001.

[10] M. Siavashi, H. Rasam, and A. Izadi, "Similarity Solution of Air and Nanofluid Impingement Cooling of a Cylindrical Porous Heat Sink," Journal of Thermal Analysis and Calorimetry, vol. 135, no. 2, pp. 1399-1415, 2019.

[11] Usman, P. Lin, A. Ghaffari, and I. Mustafa, "Similarity Solution of the Partial Differential Equations that Model Water/Magnetite Nanofluid Flow and Heat Transfer on a Stretchable Rotating Disk Subject to Thermal Radiation and Lorentz Force," Numerical Methods for Partial Differential Equations, 2020, doi: 10.1002/num.22677.

[12] O. K. Koriko and I. L. Animasaun, "New Similarity Solution of Micropolar Fluid Flow Problem Over an Uhspr in The Presence of Quartic Kind of Autocatalytic Chemical Reaction," Frontiers in Heat and Mass Transfer, vol. 8, no. 26, pp. 1-12, 2017, doi: 10.5098/hmt.8.26.

[13] M. Hasanuzzaman, B. Mandal, and M. M. T. Hossain, "A Study of Similarity Solution of Unsteady Combined Free and Force Convective Laminar Boundary Layer Flow About a Vertical Porous Surface with Suction and Blowing," Annals of Pure and Applied Mathematics, vol. 6, no. 1, pp. 85-97, 2014.

[14] Y. M. Yatim, B. R. Duffy, and S. K. Wilson, "Travelling-Wave Similarity Solutions for an Unsteady Shear-StressDriven Dry Patch in A Flowing Film,” Sains Malaysina, vol. 42, no. 7, pp. 975-980, 2013.

[15] S. S. Abas and Y. M. Yatim, "Similarity Solution for an Unsteady Dry Patch in A Shear-Stress-Driven Thin Film of Non-Newtonian Power-Law Fluid," In AIP Conference Proceedings, vol. 1605, 2014, pp. 361-366, doi: $10.1063 / 1.4887616$

[16] M. Ehrhardt and R. E. Mickens, "A Nonstandard Finite Difference Scheme for Convection-Diffusion Equations Having Constant Coefficients," Applied Mathematics and Computation, vol. 219, no. 12, pp. 6591-6604, 2013, doi: 10.1016/j.amc.2012.12.068.

[17] P. M. Jordan, "A Nonstandard Finite Difference Scheme for Nonlinear Heat Transfer in A Thin Finite Rod," The Journal of Difference Equations and Applications, vol. 9, no. 11, pp. 1015-1021, 2003, doi: 10.1080/1023619031000146922.

[18] L. Wang and L. I. Roeger, "Nonstandard Finite Difference Schemes for A Class of Generalized Convection-Diffusion-Reaction Equations," Numerical Methods for Partial Differential Equations, vol. 31, no. 4, pp. 1288-1309, 2015.

[19] L. Vulkov and A. I. Zadorin, "Two-grid Algorithms for The Solution of 2D Semilinear Singularly-perturbed Convection-diffusion Equations Using an Exponential Finite Difference Scheme," In AIP Conference Proceedings, vol. 1186, 2009, pp. 371-379, doi: 10.1063/1.3265351.

[20] A. C. Radhakrishna Pillai, "Fourth-Order Exponential Finite Difference Methods for Boundary Value Problems of Convective Diffusion Type," International Journal for Numerical Methods in Fluids, vol. 37, no. 1, pp. 87-106, 2001, doi: 10.1002/fld.167.

[21] C. Wang, "A New Way to Generate an Exponential Finite Difference Scheme for 2D Convection-Diffusion Equations," Journal of Applied Mathematics, vol. 2014, no. 3, pp. 1-14, 2014, doi: 10.1155/2014/457938.

[22] G. B. Li, L. M. Jung and G. Zhi, "The Perturbational Finite Difference (PFD) Scheme of Variational Step of Convection-Diffusion Equation," Journal of Hydrodynamics, vol. 20, no. 3, pp. 293-299, 2005.

[23] D. M. Young, "Iterative Solution of Large Linear Systems," London: Academic Press, 1971.

[24] W. Hackbusch, "Iterative Solution of Large Sparse Systems of Equations," Springer-Verlag, 1995.

[25] Y. Saad, "Iterative Methods for Sparse Linear Systems," International Thomas Publishing, 1996. 
[26] R. Rahman, N. A. M. Ali, J. Sulaiman, and F. A. Muhiddin, "Caputo's Finite Difference Solution of Fractional Two-Point Boundary Value Problems Using SOR Iteration,” In AIP Conference Proceedings, vol. 2013, 2018, pp. 020034, doi: 10.1063/1.5054233.

[27] N. A. M. Ali, R. Rahman, and J. Sulaiman, "SOR Iterative Method with Wave Variable Transformation for Solving Advection-Diffusion Equations," In AIP Conference Proceedings, vol. 2013, no. 1, 2018, doi: 10.1063/1.5054235.

[28] A. Sunarto, J. Sulaiman, and A. Saudi, "Implicit Finite Difference Solution for Time-Fractional Diffusion Equations Using AOR Method," In Journal of Physics: Conference Series, vol. 495, no. 1, 2014, doi: 10.1088/1742-6596/495/1/012032

[29] A. Sunarto, P. Agarwal, J. Sulaiman, J. V. L. Chew, and E. Aruchunan, "Iterative Method for Solving One-Dimensional Fractional Mathematical Physics Model Via Quarter-Sweep and PAOR," Advances in Difference Equations, vol. 2021, pp. 1-12, 2021.

[30] I. K. Youssef and A. A. Taha, "On Modified Successive Overrelaxation Method," Applied Mathematics and Computation, vol. 219, no. 9, pp. 4601-4613, 2013, doi: 10.1016/j.amc.2012.10.071.

[31] F. A. Muhiddin, J. Sulaiman, and A. Sunarto, "Numerical Evaluation of Quarter-Sweep KSOR Method to Solve Time-Fractional Parabolic Equations," SSRG International Journal of Engineering Trends and Technology, vol. 1, pp. 63-69, 2020, doi: 10.14445/22315381/CATI2P210.

[32] D. J. Evans, "Group Explicit Iterative Methods for Solving Large Linear Systems," International Journal of Computer Mathematics, vol. 17, pp. 81-108, 1985, doi: 10.1080/00207168508803452.

[33] A. Saudi and J. Sulaiman, "Path Planning for Mobile Robot Using 4EGSOR Via Nine-Point Laplacian (4EGSOR9L) Iterative Method," International Journal of Computer Applications, vol. 53, no. 16, pp. 38-42, 2012, doi: $10.5120 / 8509-2568$.

[34] M. K. M. Akhir, M. Othman, J. Sulaiman, and Z. A. Majid, "Four Point Explicit Decoupled Group Iterative Method Applied to Two-Dimensional Helmholtz Equation," International Journal of Mathematical Analysis, vol. 6, no. 20, pp. 963-974, 2012.

[35] J. Sulaiman, M. Othman, and M. K. Hasan, "Nine Point-EDGSOR Iterative Method for the Finite Element Solution of 2D Poisson Equations," In Conference: Proceedings of the International Conference on Computational Science and Its Applications: Part I, 2009, doi: 10.1007/978-3-642-02454-2_59

[36] N. A. M. Ali, R. Rahman, J. Sulaiman, and K. Ghazali, "Numerical Solutions of Unsteady Advection-Diffusion Equations by Using EG Iteration with Wave Variable Transformation," In Journal of Physics: Conference Series, vol. 1358, 2019, doi: 10.1088/1742-6596/1358/1/012049.

[37] T. K. Bee, N. M. Ali, and L. C. Hong, "Explicit Group Methods in The Solution of the 2-D Convection-Diffusion Equations," In Proceedings of the World Congress on Engineering, vol. 3, 2010.

[38] M. Alquran, "Solitons and Periodic Solutions to Nonlinear Partial Differential Equations by The Sine-Cosine Method," Applied Mathematics and Information Sciences, vol. 6, no. 1, pp. 85-88, 2012.

[39] A. M. Wazwaz, "Travelling Wave Solutions for Combined and Double Combined Sine-Cosine-Gordon Equations by The Variable Separated ODE Method," Applied Mathematics and Computation, vol. 177, no. 2, pp. 755-760, 2006, doi: 10.1016/j.amc.2005.09.104.

[40] R. C. Mittal and A. Tripathi, "Numerical Solutions of Two-Dimensional Unsteady Convection-Diffusion Problems Using Modified Bi-Cubic B-Spline Finite Elements," International Journal of Computer Mathematics, vol. 94, no. 1, pp. 1-21, 2017, doi: 10.1080/00207160.2015.1085976.

[41] R. C. Mittal, S. Kumar, and R. Jiwwari, "A Cubic B-Spline Quasi-Interpolation Method for Solving Two-Dimensional Unsteady Advection Diffusion Equations," International Journal of Numerical Methods for Heat \& Fluid Flow, vol. 30, no. 9, pp. 4281-4306, 2020, doi: 10.1108/HFF-07-2019-0597.

[42] H. W. Sun and L. Z. Li, "A CCD-ADI Method for Unsteady Convection-Diffusion Equations," Computer Physics Communications, vol. 185, no. 3, pp. 790-797, 2014, doi: 10.1016/j.cpc.2013.11.009. 